This is the author's final, peer-reviewed manuscript as accepted for publication. The publisher-formatted version may be available through the publisher's web site or your institution's library.

\title{
Genome-wide association analysis identified SNPs closely linked to a gene resistant to Soil-borne wheat mosaic virus
}

Shubing Liu, Xiping Yang, Dadong Zhang, Guihua Bai, Shiaoman Chao, William Bockus

\section{How to cite this manuscript}

If you make reference to this version of the manuscript, use the following information:

Liu, S., Yang, X., Zhang, D., Bai, G., Chao, S., \& Bockus, W. (2014). Genome-wide association analysis identified SNPs closely linked to a gene resistant to Soil-borne wheat mosaic virus. Retrieved from http://krex.ksu.edu

\section{Published Version Information}

Citation: Liu, S., Yang, X., Zhang, D., Bai, G., Chao, S., \& Bockus, W. (2014). Genomewide association analysis identified SNPs closely linked to a gene resistant to Soilborne wheat mosaic virus. Theoretical and Applied Genetics, 127(5), 1039-1047.

Copyright: @ Springer-Verlag Berlin Heidelberg (outside the USA) 2014

Digital Object Identifier (DOI): doi:10.1007/s00122-014-2277-z

Publisher's Link: http://link.springer.com/article/10.1007\%2Fs00122-014-2277-z

This item was retrieved from the K-State Research Exchange (K-REx), the institutional repository of Kansas State University. K-REx is available at http://krex.ksu.edu 
Genome-wide association analysis identified SNPs closely linked to a gene resistant to Soil-borne wheat mosaic virus

Shubing Liu $\bullet$ Xiping Yang • Dadong Zhang $\bullet$ Guihua Bai* ${ }^{*}$ Shiaoman Chao $\bullet$ William Bockus

Shubing Liu $\bullet$ Xiping Yang • Dadong Zhang • Guihua Bai

Department of Agronomy, Kansas State University, Manhattan, KS 66506, USA

Guihua Bai *

USDA-ARS, Hard Winter Wheat Genetics Research Unit, 4008 Throckmorton Hall, Manhattan, KS 66506, USA

Email: guihua.bai@ars.usda.gov, gbai@ksu.edu, phone:785-532-1124

Shiaoman Chao

USDA-ARS, Cereal Crops Research Unit, Fargo, ND, USA

William Bockus

Department of Plant Pathology, Kansas State University, Manhattan, KS 66506, USA

Author Contributions: GB designed experiments, DZ, XY, SC, SL and GB conducted experiments, SC, WB, GB contributed reagents, DZ, XY and SL performed data analysis, XY, SL, and GB wrote the manuscript.

Key Message: Using association and linkage mapping, two SNP markers closely linked to the SBWMV resistance gene on chromosome 5D were identified and can be used to select the gene in breeding.

Conflict of Interest:

The authors declare that they have no conflict of interest. 


\begin{abstract}
Soil-borne wheat mosaic virus (SBWMV) disease is a serious viral disease of winter wheat growing areas worldwide. SBWMV infection can significantly reduce grain yield up to $80 \%$. Developing resistant wheat cultivars is the only feasible strategy to reduce the losses. In this study, wheat Infinium iSelect Beadchips with 9K wheat SNPs were used to genotype an association mapping population of 205 wheat accessions. Six new SNPs from two genes were identified to be significantly associated with the gene for SBWMV resistance on chromosome 5D. The SNPs and Xgwm469, a SSR marker that has been reported to be associated with the gene, were mapped close to the gene using $\mathrm{F}_{6}$-derived recombinant inbred lines (RILs) from the cross between a resistant parent 'Heyne' and a susceptible parent 'Trego'. Two representative SNPs, wsnp_CAP11_c209_198467 and wsnp_JD_c4438_5568170, from the two linked genes in wheat were converted into KBioscience Competitive Allele-Specific Polymerase (KASP) assays and can be easily used in marker-assisted selection to improve wheat resistance to SBWMV in breeding.
\end{abstract}

Keywords: SBWMV resistance·Association mapping $\cdot$ Triticum aestivum $\cdot$ Polymyxa graminis $9 \mathrm{~K}$ wheat SNP chip· KASP marker 
Introduction

Soil-borne wheat mosaic virus (SBWMV) causes an important disease in winter wheat growing areas worldwide (Barbosa et al. 2001; Hariri et al. 1987; Kapooria et al. 2000; Koenig and Huth 2003; Lebas et al. 2009; Sawada 1927). In the U. S., it is a serious winter wheat disease in the Great Plains and eastern U. S. winter wheat growing regions (Cadle-Davidson et al. 2006). SBWMV is a member in Furovirus genus, and can be transmitted to wheat roots by the plasmodiophorid vector Polymyxa graminis (Zhang et al. 2011). SBWMV-infected plants show yellow to light green leaves with dark green mottling, stunting, reduced tillers, and low kernel and test weights (Koehler et al. 1952). Reported yield losses due to SBWMV infection range from $10 \%$ to $30 \%$ and, in extreme cases up to $80 \%$ in seriously infected fields in the U. S. and 50\% in Brazil (Bever and Pendleton 1954; Hao et al. 2012; Myers et al. 1993).

SBWMV infects wheat in wet soil at $15^{\circ} \mathrm{C}$ to $20^{\circ} \mathrm{C}$ with an optimum temperature at $17^{\circ} \mathrm{C}$ (Driskel et al. 2002; Shirako et al. 2000). Infected plants usually show disease symptoms in early spring, and cool spring temperatures promote the development of the symptoms. Symptoms stop developing on leaves when the average temperature exceeds $20^{\circ} \mathrm{C}$, but can be reinitiated on newly emerged leaves given conductive weather conditions (Cadle-Davidson et al. 2006). Cultural practices including crop rotation, delayed drilling, application of fungicides, and soil treatments have been proposed as possible control measures to reduce SBWMV damage, but they have proven neither practical, nor effective (Bass et al. 2006; Johnson 1942; McKinney 1923). Growing resistant cultivars is the only economical method to reduce the disease losses (Barbosa et al. 2001; Merkle and Smith 1983; Modawi et al. 1982). Resistant cultivars usually have very low incidence of symptomatic plants, but not necessarily low severity once infected (Myers et al. 1993; CadleDavidson et al. 2006). 
Many studies have been conducted to identify resistance sources in wheat and investigate genetic bases of wheat resistance to SBWMV (Barbosa et al. 2001; Merkle and Smith 1983; Miyake 1938; Modawi et al. 1982; Shaalan et al. 1966); one to three genes for SBWMV resistance have been documented (Shaalan et al. 1966; Merkle and Smith 1983; Modawi et al. 1982). Using linkage mapping, a single QTL conferring SBWMV resistance was mapped independently on the long arm of wheat chromosome 5D (5DL) of the hard red winter wheat 'Karl 92' (Narasimhamoorthy et al. 2006), 'Pioneer 26R61', 'AGS 2020' (Hao et al. (2012), and 'KS96WGRC40' derived from Aegilops tauschii (Hall et al. 2009). Interestingly, one gene, Sbm1, conferring resistance to Soil-borne cereal mosaic virus (SBCMV) from three wheat cultivars, Cadenza, Tremie and Claire, was also mapped in the similar region to that for SBWMV resistance on chromosome 5DL (Bass et al. 2006; Perovic et al. 2009). Another major QTL (QSbm.ubo-2BS) for SBCMV resistance was mapped to short arm of chromosome 2B (Maccaferri et al. 2011).

In recent years, association studies have been extensively used to discover and validate quantitative trait loci (QTLs) or genes for important traits and to map candidate genes in many crop species (Kump et al. 2011; Huang et al. 2010). Contrasting with linkage mapping, association mapping has the capability to exploit recombination events over multiple breeding cycles (Myles et al. 2009; Zhu et al. 2008) without the need for developing new mapping populations, thus it has been used successfully to identify genes or QTLs in many plant species including Arabidopsis (Atwell et al. 2010), rice (Huang et al. 2010), maize (Krill et al. 2010; Kump et al. 2011) and potato (Malosetti et al. 2007). In wheat, association analysis has also been successfully used to identify new genes for resistance to stem rust and pre-harvest sprouting, and for quality traits (Letta et al. 2013; Kulwal et al. 2012; Reif et al. 2011). Previously, we have identified a SSR marker, Xgwm469 that was associated with the SBWMV resistance gene on 5DL after analyzing 282 SSR markers across 205 wheat accessions (Zhang et al. 2011). To further fine map the gene, the current study genotyped the same set of wheat accessions using Infinium iSelect Beadchips with 9K wheat SNPs (Cavanagh et al. 2013) 
to i) identify SNP markers that are highly associated with the resistance gene, ii) validate the linkage between the identified markers and the resistance gene by linkage mapping, iii) identify the syntenic regions in rice, barley and Brachypodium for further comparative fine mapping and map-based cloning of the gene, and (iv) develop breeder-friendly KBioscience Competitive Allele-Specific Polymerase chain reaction (KASP) assays for tightly linked SNPs to the gene for marker-assisted selection (MAS) in breeding.

\section{Materials and methods}

Plant materials. The association mapping population including 205 wheat accessions with 137 hard winter wheat (HWW) and 68 soft winter wheat (SWW) from six 2008 HWW and SWW nurseries (Zhang et al. 2010). Seeds for DNA isolation and disease evaluation were originated from a single plant of each accession increased in a greenhouse to minimize within-line heterogeneity. All these accessions were genotyped using Infinium iSelect Beadchips with 9K wheat SNPs (Cavanagh et al. 2013).

An $\mathrm{F}_{6}$ RIL population with 93 RILs was used to map the SBWMV resistance gene and the associated SNP and SSR markers. The population was developed from the cross 'Trego' $\mathrm{x}$ 'Heyne' by single-seed descent (Zhang et al 2012). 'Heyne' (PI 612577) is an SBWMV-resistant HWW cultivar, whereas 'Trego' (PI 612576) is a highly SBWMV-susceptible HWW cultivar.

Disease evaluation and statistical analysis. All 205 wheat accessions were evaluated for SBWMV resistance in an SBWMV- infested field at the Rocky Ford Research Farm of Kansas State University, Manhattan, KS in 2009-2010 and 2010-2011 wheat growing seasons. The nursery has shown consistent and severe SBWMV infection on susceptible wheat cultivars from 2006 to 2009. Each experiment had two replicates and each accession was planted in a 3-ft row plot. After planting, the nursery was irrigated daily till seedling fully emerged to initiate the virus infection. A previously 
described 1 to 4 rating scale (Modawi et al. 1982; Hall et al. 2009) was used to evaluate disease damage twice with a 2-wk interval between the tillering and jointing stages (Feekes growth stage 3-6) according to overall leaf symptoms in each row: 1 or resistant $(\mathrm{R})$ represents no mottling on the leaves and no stunting of plants; 2 or moderately resistant (MR) represents very slight mottling and no stunting; 3 or moderately susceptible (MS) represents obvious mottling with some stunting; and 4 or susceptible (S) represents severe mottling and stunting. RILs of 'Trego' $\mathrm{x}$ 'Heyne' were evaluated for SBWMV resistance in the same field nursery from 2010 to 2012 growing seasons using the same rating scale described for association mapping.

Variance analysis was conducted using GLM in SAS for windows v9 (SAS Institute, Inc., Cary, NC) to determine the effects of genotypes (G), environments (E), and $\mathrm{G} x \mathrm{E}$ interactions on SBWMV resistance. The heritability $\left(h^{2}\right)$ was estimated using the formula $\mathrm{H}^{2}=\sigma_{\mathrm{g}}^{2} /\left(\sigma_{\mathrm{g}}^{2}+\sigma_{\mathrm{ge}}^{2} / \mathrm{n}+\right.$ $\sigma_{\mathrm{e}}^{2} \mathrm{enr}$ ) based on the data from variance analysis, where $\sigma_{\mathrm{g}}^{2}$ is the variance among wheat lines, $\sigma_{\mathrm{ge}}^{2}$ is the variance for $\mathrm{G} \times \mathrm{E}, \sigma_{\mathrm{e}}^{2}$ is the variance of environments, $\mathrm{n}$ is the number of environments, and $\mathrm{r}$ is the number of replicates (Toojinda et al., 1998).

DNA extraction, 9K SNP assay and SSR marker genotyping. The 205 accessions were genotyped using the 9K Infinium iSelect Beadchips as described by Cavanagh et al. (2013). A total of 6985 single nucleotide polymorphism (SNP) markers were polymorphic in the association population. The RIL population was genotyped with two representative SNPs, wsnp_CAP11_c209_198467 and wsnp_JD_c4438_5568170, for two genes linked to the SBWMV resistance gene using KASP assay, with the marker Xgwm469 that was identified to be linked to the SBWMV resistance gene in the previous study, and an additional 37 simple sequence repeat (SSR) markers mapped on chromosome 5D. Polymorphic markers between the two parents were run in the RIL population to construct a 5D linkage map. Procedures for tissue collection, DNA extraction and SSR analysis were described previously (Liu et al. 2008). 


\section{Population structure, linkage disequilibrium and association analysis}

Population structure was assessed by Structure 2.3.3 (Pritchard et al. 2000) using a set of 282 informative SSR markers. Those markers were selected from a set of 2000 wheat SSR markers and are evenly distributed across the wheat genome according to our previous screening results and published information (Zhang et al. 2010). The admixture model was used for structure analysis and the number of sub-populations $(\mathrm{K})$ was set as 1 through 10 with variable length of burn-in period and number of iterations at $20,000-250,000$.

Genotypic data of seven SSR markers on 5D (Somers et al. 2004), Xwmc233, Xgwm358, Xgdm138, Xgwm182, Xcfd10, Xgwm469, Xgwm565, and the significant SNP markers associated with SBWMV resistance on 5D for the 205 accessions were used to calculate the linkage disequilibrium by TASSEL (Bradbury et al. 2007).

Association analyses using the genotypic data generated from the Infinium iSelect Beadchips were conducted separately for the phenotypic data collected in 2010 and 2011 following the method described in Zhang et al. (2011). Xgwm469 was also included in association mapping. To minimize false-positive associations caused by rare alleles, all alleles with a frequency lower than $5 \%$ were excluded. All analyses were conducted using PROC MIXED in SAS (ver. 9.1.2; SAS Institute Inc., Cary, NC). A threshold of $P<10^{-6}$ was obtained using the experiment-wise association significance probability obtained through 10,000 permutation tests implemented in TASSEL to claim alleles that were significantly associated with SBWMV resistance (Letta et al. 2013).

\section{Linkage mapping}

A linkage map was constructed for selected polymorphic SNPs and SSR markers on 5D, and the SBWMV resistance phenotypic data generated from the RIL population using JoinMap ver. 3.0 (Van Ooijen and Voorrips 2001). Recombination fractions were converted into centiMorgans (cM) using the Kosambi function (Kosambi 1944). The threshold of logarithm of odd (LOD) score was set 
at 3.0 to claim linkage between markers with 0.4 as a maximum fraction of recombination. The goodness-of-fit between observed and expected segregation ratios between two alleles was analyzed for each marker locus using a chi-square-test.

\section{BLAST analysis}

Sequences containing SNPs that were significantly associated with SBWMV resistance were used as queries to search in the Chinese Spring shot-gun genome sequencing database (Brenchley et al. 2012) using BLASTN, and the identified sequences were used as queries to search for the syntenic region in barley, Brachypodium and rice by blasting their corresponding genome databases (www.barley.org; http://www.phytozome.org; http://www.jcvi.org). A significant match was declared when at least half of the queried sequences, but not fewer than 300 bases, showed at least $70 \%$ nucleotide identity with an e-value lower than $\mathrm{e}^{-20}$.

\section{Conversion of SNP markers to KASP assay}

Sequences harboring the SNPs that were significantly associated with SBWMV resistance were selected to develop KASP assays. The KASP assays were validated on the two parents of the RIL population and the 205 wheat accessions used for association mapping following the instructions from the manufacturer (http://www.kbioscience.co.uk/reagents/KASP_manual.pdf). KASP primer sequences CACGCCATTAGCAGACGTACGTA-FAM, and ACGCCATTAGCAGACGTACGTG-HEX are two forward primers, and GGGGAGTTCCCGTGTATATGTAAATAAAT is the reverse primer for wsnp_CAP11_c209_198467; and GCCATCAGAAGTATGGGCGACT-FAM and CCATCAGAAGTATGGGCGACC-HEX are two forward primers, and AAATGACTGGTCATCACCTTGTATCCTT is a reverse primer for wsnp_JD_c4438_5568170. 
A $6 \mu 1$ reaction of KASP assay includes $3 \mu 1$ of 2x reaction mix, $0.106 \mu l$ of assay mix (LGC Genomics, Beverly, MA) and $3 \mu \mathrm{l}$ of genomic DNA at $15 \mathrm{ng} / \mu \mathrm{l}$. PCR and fluorescent endpoint readings were carried out using an ABI 7900HT Real-Time PCR System (Life Technology, Grand Island, NY). PCR thermal cycling profile followed the manufacturer's manual (http://www.kbioscience.co.uk/reagents/KASP_manual.pdf).

\section{Results}

SBWMV resistance in the association mapping and linkage mapping populations. About $70 \%$ of the accessions in the association mapping population were either resistant or moderately resistant, and about 30\% were moderately susceptible or highly susceptible in both years (Fig. 1A), indicating the majority of US winter wheat breeding lines contain a resistance gene to SBWMV. A high correlation coefficient $(0.85, P<0.01)$ was observed between the two years of disease data, suggesting a high repeatability between the field tests. The heritability of the SBWMV resistance was high $(88.03 \%)$ in this population. However, fewer highly resistant accessions were observed in the 2010 experiment than the 2011 experiment, although the total number of resistant (MR and R) accessions remained the same in both years, indicating that environmental factors affected the expression level of SBWMV resistance.

In the 'Trego' $\mathrm{x}$ 'Heyne' RIL population, SBWMV infection data were also highly repeatable among the three years with correlation coefficients ranging from 0.82 to $0.88(P<0.01)$ with a high heritability of $93.2 \%$. The distribution of SBWMV disease scores for the “'Trego' $\mathrm{x}$ 'Heyne' RILs deviated significantly from the normal distribution, and showed a bimodal distribution with two peaks towards resistant and susceptible parents, respectively (Fig. 1B). The results suggest that a major gene may be involved in SBWMV resistance in 'Heyne'.

\section{SNP markers for the SBWMV resistance gene}


Genotyping the association mapping population with the 9K Infinium iSelect Beadchips identified 6985 polymorphic SNP markers. Association analysis showed that six SNPs, wsnp_CAP11_c209_198467, wsnp_CAP11_c209_198671, wsnp_CAP11_c209_198432, wsnp_JD_c4438_5567972, wsnp_JD_c4438_5568170 and wsnp_JD_c4438_5567834 were significantly associated with resistance in both years $\left(P<10^{-40}\right)$ (Fig. 2). BLASTN analysis based on comparison with the Chinese Spring whole genome sequence (Brenchley et al. 2012) found that a wheat contig, $\operatorname{ctg} 120561$, contains sequences of three SNPs, wsnp_CAP11_c209_198467, wsnp_CAP11_c209_198671 and wsnp_CAP11_c209_198432, and this contig is homologous to a Barke_contig_280231 on barley chromosome 5HL, one rice gene, Os03g62780, and a Brachypodium gene, Bd1g01880, encoding a RNA binding domain containing protein (Fig. 3). Association analysis showed that these three SNPs had the highest significant associations with the resistance. Sequences of wsnp_JD_c4438_5567972, wsnp_JD_c4438_5568170 and wsnp_JD_c4438_5567834 were found in the wheat contig ctg403927 that is homologous to a Barke_contig_513419 on barley chromosome 5HL, a rice gene Os03g30890 and a Brachypodium gene, Bd4g43690 encoding a putative kinase family protein (Fig. 3). Three SNPs in each of two genes that showed significant association with SBWMV resistance suggest that the two genes are closely linked to the SBWMV resistance gene, or maybe the candidate genes for SBWMV resistance.

A previously reported marker, Xgwm469 for SBWMV resistance on chromosome 5D (Zhang et al. 2011), was also significant in this study. LD analysis indicated that Xgwm469 had significant LD with all the six SNPs that were significantly associated with SBWMV resistance (Supplemental Fig. 1), while the other two markers, Xgwm565 and Xcfd10 on 5D that flanked Xgwm469 at 20 centimorgans (cM) apart, and the other four markers (Xwmc233, Xgwm358, Xgdm138, and Xgwm182) on 5D showed no LD with the six SNPs. The results indicated that the six SNPs are closer to Xgwm469, than to the other SSRs analyzed. 


\section{Linkage mapping}

To validate the associations detected among the SBWMV resistance gene, and previously reported SSR marker Xgwm469, and the 6 SNPs identified in this study, 37 SSR markers on 5D were genotyped across 93 RILs derived from 'Trego' x 'Heyne' cross. Because the six SNPs are from two wheat genes, only one SNP from each gene, wsnp_CAP11_c209_198467 and wsnp_JD_c4438_5568170, was used for linkage analysis. A 5D linkage map was constructed with six SSR and two SNP markers that spanned $63 \mathrm{cM}$. The two representative SNPs and one SSR, Xgwm469, were all closely linked to the SBWMV resistance gene within $15 \mathrm{cM}$ in the linkage group, which validated the result of LD analysis. The resistant gene, designated as Sbwm1, is located $2 \mathrm{cM}$ away from wsnp_CAP11_c209_198467 and flanked by Xgwm272 and wsnp_CAP11_c209_198467 (Fig. 3A).

\section{KASP assay for selecting Sbwm1}

To develop breeder friendly markers for marker-assisted selection, the two selected SNPs, wsnp_CAP11_c209_198467 and wsnp_JD_c4438_5568170, were converted to KASP assays and genotyped the same 205 accessions used for association mapping (Supplemental Fig. 2). The results showed that the genotypic data obtained from the KASP assays matched with the data based on the Infinium assay. Four haplotypes were identified for the two SNPs across the 205 accessions (Table 1) with two haplotypes associated with resistant accessions (R1-R2) and two associated with susceptible accessions (S1-S2). For wsnp_CAP11_c209_198467, A and G allele presents in all of the resistant and susceptible accessions, respectively. For wsnp_JD_c4438_5568170, A allele present in $96.5 \%$ of the resistant and $\mathrm{G}$ allele present in $95 \%$ of the susceptible accessions, the remaining accessions conferring $\mathrm{G}$ and $\mathrm{A}$ allele in the resistant and susceptible accessions. There are some accessions conferring A allele but susceptible to SBWM and some accession conferring G allele but 
resistant (Table 1). Thus, the two KASP assays can effectively distinguish resistant and susceptible alleles in the diverse wheat panel and can be used for selection of Sbwm1 in breeding programs.

\section{Discussion}

Environmental factors such as soil water content after planting and spring temperature, etc. affect expression of SBWMV symptoms in the field. In this study, a slight difference in disease ratings between two years was observed for some cultivars. For both mapping populations, more accessions were in the highly resistant category within the resistant response in 2011 (association mapping population) and 2012 (linkage mapping population) than in 2010 experiment (Fig. 1). This could be attributed to the environmental effects that affect the expression levels of wheat resistance to SBWMV in different years. Given the spatial patchiness of virus or vector as well as the environmental sensitivity of transmission and symptom expression, reliable evaluation of resistance requires compilation of multiple years of replicated data (Cadle-Davidson et al. 2006). However, a high correlation coefficient was observed for phenotypic data collected between different years (0.82-0.88) in both the association and linkage mapping studies, and the rating changes mainly occurred within the resistant or susceptible categories, not across between the two categories, and the heritability was high in the both RIL and association mapping populations, thus the field nursery used in this study provided highly repeatable phenotypic data for mapping work.

Several independent linkage-mapping studies have identified a major locus for SBWMV resistance on the long arm of chromosome 5D (Narasimhamoorthy et al. 2006; Hao et al. 2012; Hall et al. 2009). Our previous association mapping work identified a SSR marker, Xgwm469 that was associated with Sbwm1 (Zhang et al. 2011). In the current study, we conducted a genome-wide association study using the same set of wheat accessions and Infinium iSelect Beadchips with 9K wheat SNPs (Cavanagh et al. 2013), and successfully identified six new SNPs that were significantly associated with Sbwm1. Homology searching in the wheat genome sequencing database (Brenchley 
et al. 2012) reveals that the six SNPs belong to two genes with three SNPs in each of the wheat genes. The two sets of SNPs showed significant LD with Xgwm469, but not with two SSR markers Xcfd10 and Xgwm565 in this region and other markers on the chromosome (Supplemental Fig. 1), suggesting that Xgwm469 and the newly identified SNPs closely link to Sbwm1 on 5D. Further linkage mapping in the 'Trego' $\mathrm{x}$ 'Heyne' population validated this prediction because $\mathrm{Xgwm} 469$ is the closest marker to the two mapped SNP markers (Fig3. A), Xcfd10 and Xgwm565 are far away from Sbmv-1, LD decayed due to the longer distance between Sbmv-1 and these markers.

To further investigate the genetic relationship between these SNP-containing genes and Sbwm1, the $\mathrm{F}_{6}$ RIL population of 'Trego' $\mathrm{x}$ 'Heyne' was used to construct a linkage map, and the two representative SNPs and Xgwm469 were all mapped near Sbwm1 in the same linkage group corresponding to chromosome 5D (Fig. 3). Sbwm1 was flanked by Xgwm272 and wsnp_CAP11_c209_198467 with wsnp_CAP11_c209_198467 as the closest marker that was 2 cM from Sbwm1. Therefore, using combined association mapping and linkage mapping together, we validated the trait and marker association, successfully determined the genetic distance between the markers and Sbwm1, and laid solid ground for map-based cloning of Sbwm1.

Sbm1 for SBCMV resistance was also reported in the distal region of chromosome arm 5DL (Bass et al. 2006). Symptoms of SBCMV infection are similar to those caused by SBWMV, but they only share $\sim 70 \%$ sequence identity, thus were classified as different species (Diao et al. 1999; Koenig et al 2003). Xgwm469 was reported to co-segregate with SBCMV resistance (Perovic et al. 2009; Bass et al. 2006), whereas a SBWMV resistance QTL was mapped independently on the similar chromosome region in different winter wheat cultivars (Narasimhamoorthy et al. 2006, Hao et al. 2012) and an Aegilops tauschii-derived germplasm line (Hall et al. 2009). Comparison of locations of common markers closely linked to the resistance gene between our map and these maps from other studies (Narasimhamoorthy et al. 2006, Hall et al. 2009; Perovic et al. 2009) suggests that 
the genes for SBWMV and SBCMV resistance mapped on 5DL in different studies are likely the same gene.

In wheat, most association mapping studies used SSR and DArT markers (Kulwal et al. 2012; Letta et al 2012; Reif et al. 2011; Zhang et al. 2011). However, only limited numbers of these markers are available and they are not suitable for high-throughput assay (SSR) or used in routine breeding programs (DArT). SNP is the most abundant class of polymorphic markers in a genome and has become a powerful tool in association studies in different crops (Huang et al. 2010; Kump et al. 2011). The development of wheat Infinium iSelect Beadchips greatly facilitates the high throughput genome-wide association analysis of wheat accessions (Cavanagh et al. 2013). In this study, the association mapping panel was genotyped using the 9K SNP chips and 6895 SNPs were found polymorphic. One set of closely linked SNPs were identified only at 2 cM away from Sbwm1, instead of the markers $14 \mathrm{cM}$ away from the gene identified in a previous study (Fig. 3), which demonstrated the power of combined genome-wide association mapping and linkage mapping to identify closely linked markers for important traits using the wheat Infinium iSelect Beadchips. These identified SNPs will facilitate marker-assisted selection, fine mapping, and cloning of Sbwm1.

Because it is cost ineffective to directly use the SNP chip in breeding selection, breeder friendly SNP markers for Sbwm1 will facilitate deployment of this gene into elite breeding line. KASP assay is a time saving and cost-effective genotyping assay for single SNP analysis (Allen et al. 2011; Terracciano et al. 2013; Semagn et al. 2013). The competitive allele specific PCR method implemented in the KASP assay has been recently developed (Nijman et al. 2008) and applied successfully in polyploids such as wheat (Allen et al. 2011) and cotton (Byers et al. 2012), thus demonstrating its usefulness as a SNP genotyping platform for marker-assisted breeding. In this study, two significant SNP markers, wsnp_CAP11_c209_198467 and wsnp_JD_c4438_5568170, representing two genes closely linked to $S b w m 1$ were converted to the KASP assays. The genotypic data of the two SNPs (KASP-wsnp_CAP11_c209_198467, KASP-wsnp_JD_c4438_5568170) 
derived from KASP assays coincided with the original 9K SNP data generated from the associationmapping panel (Supplemental Fig. 2).

For wsnp_CAP11_c209_198467, the A and G allele was identical with SBWM resistance among the 205 association mapping accessions, while for wsnp_JD_c4438_5568170, A and G allele only presents in most the resistant and susceptible accessions, the remaining accessions conferring $\mathrm{G}$ and A allele in the resistant and susceptible accessions (Table 1), which indicated that wsnp_CAP11_c209_198467 was closer to Sbwm1 than wsnp_JD_c4438_5568170 and more effective than wsnp_JD_c4438_5568170 for MAS of Sbwm1. This result was confirmed by linkage mapping in the 'Trego' $\mathrm{x}$ 'Heyne' population, in which wsnp_CAP11_c209_198467 is mapped as the nearest marker to Sbwm1. Therefore, KASP-wsnp_CAP11_c209_198467 is the best marker for MAS of Sbwm1 and KASP-wsnp_JD_c4438_5568170 is also an useful marker for Sbwm1 in case no polymorphism of wsnp_CAP11_c209_198467. We have extensively used the SNPs for transfer of Sbwm1 into breeding materials from the Great Plains to improve SBWMV resistance.

Comparative mapping with barley, rice and Brachypodium determined that the six wheat SNPs in two wheat contigs (ctg120561 and ctg403927) are homologous to two chromosome regions each on rice chromosome 3 and on Brachypodium chromosomes 1 and 4. In wheat, the two contigs are closely linked, but they are far from each other in rice and even in different chromosomes in Brachypodium (Fig. 3), which suggests that the synteny of this region harboring Sbwm1 among wheat, rice and Brachypodium is complicated, and a comparative mapping approach using the rice and Brachypodium as reference genomes may not help much in fine mapping of the gene, however, we identified two homologous contigs on barley 5HL by blasting the barley genome sequence, suggesting the synteny between wheat and barley is better than rice and Brachypodium. Further fine mapping using the barley draft sequence, the T. tauschii physical map and the wheat draft genome database may facilitate further fine mapping and map-based cloning of this gene. 


\section{Acknowledgments}

This is contribution number 14-056-J from the Kansas Agricultural Experiment Station, Manhattan, KS, USA. This project is partly funded by the National Research Initiative Competitive Grants CAP project 2011- 68002-30029 from the USDA National Institute of Food and Agriculture. Mention of trade names or commercial products in this article is solely for the purpose of providing specific information and does not imply recommendation or endorsement by the U.S. Department of Agriculture. USDA is an equal opportunity provider and employer.

\section{Conflict of Interest.}

The authors declare that they have no conflict of interest.

\section{References}

1. Allen AM, Barker GL, Berry ST, Coghill JA, Gwilliam R, et al.(2011) Transcriptspecific, single nucleotide polymorphism discovery and linkage analysis in hexaploid bread wheat (Triticum aestivum L.). Plant Biotechnol J 9:1086-1099

2. Atwell S, Huang YS, Vilhjalmsson BJ, Willems G, Horton M, et al. (2010) Genome-wide association study of 107 phenotypes in Arabidopsis thaliana inbred lines. Nature 465:627631.

3. Barbosa M, Goulart L, Prestes A, Juliatti F (2001) Genetic control of resistance to Soilborne wheat mosaic virus in Brazilian cultivars of Triticum aestivum L. Thell. Euphytica 122: $417-422$.

4. Bass C, Hendley R, Adams MJ, Hammond-Kosack KE, Kanyuka K (2006) The Sbm1 locus conferring resistance to Soil-borne cereal mosaic virus maps to a gene-rich region on 5DL in wheat. Genome 2006 49:1140-1148. 
5. Bever WM, Pendleton JW (1954) The effect of soil-borne wheat mosaic on yield of winter wheat. Plant Dis Rep 38:266-267

6. Bradbury PJ, Zhang Z, Kroon DE, Casstevens TM, Ramdoss Y, Buckler ES (2007) TASSEL: software for association mapping of complex traits in diverse samples. Bioinformatics 23:2633-2635.

7. Brenchley R, Spannagl M, Pfeifer M, Barker GLA, D’Amore R, Allen AM, et al. (2012) Analysis of the bread wheat genome using whole-genome shotgun sequencing. Nature 491:705710

8. Byers RL, Harker DB, Yourstone SM, Maughan PJ, Udall JA (2012) Development and mapping of SNP assays in allotetraploid cotton. Theor Appl Genet 124:1201-1214

9. Cadle-Davidson L, Sorrells ME, Gray SM, Bergstrom GC (2006) Identification of small grains genotypes resistant to Soil-borne mosaic virus. Plant Dis 90:1039-1044

10. Cavanagh CR, Chao S, Wang S, Huang BE, Stephen S, et al. (2013) Genome-wide comparative diversity uncovers multiple targets of selection for improvement in hexaploid wheat landraces and cultivars. PNAS 110:8057-8062

11. Diao A, Chen J, Gitton F, Antoniw JF, Mullins J, Hall AM, and Adams MJ (1999) Sequences of European Wheat mosaic virus and Oat golden stripe virus and genome analysis of the genus Furovirus. Virology 261:331-339.

12. Driskel BA, Hunger RM, Payton ME and Verchot-Lubicz J (2002) Response of hard red winter wheat to Soil-borne wheat mosaic virus using novel inoculation methods. Phytopathology 92:347-54.

13. Hall MD, Brown-Guedira G, Klatt A, Fritz AK (2009) Genetic analysis of resistance to Soilborne wheat mosaic virus derived from Aegilops tauschii. Euphytica 169:169-176. 
14. Hao Y, Wang Y, Chen Z, Bland D, Li S, Brown-Guedira G, Johnson J (2012) A conserved locus conditioning Soilborne wheat mosaic virus resistance on the long arm of chromosome 5D in common wheat. Mol Breed 30:1453-1464

15. Hariri D, Courtillot M, Zaoui P, Lapierre H (1987) Multiplication of Soil-borne wheat mosaic virus (SBWMV) in wheat roots infected by a soil carrying SBWMV and wheat yellow mosaic virus (WYMV). Agronomie 7:789-796

16. Huang X, Wei X, Sang T, Zhao Q, Feng Q, et al. (2010) Genome-wide association studies of 14 agronomic traits in rice landraces. Nat Genet 42:961-967

17. Johnson F (1942) Heat inactivation of wheat mosaic virus in soils. Science 95:610

18. Kapooria RG, Ndunguru J, Clover GRG (2000) First reports of Soilborne wheat mosaic virus and Wheat spindle streak mosaic virus in Africa. Plant Dis 84:921

19. Koehler B, Bever WM, Bonnett OT (1952) Soilborne wheat mosaic. University of Illinois Agricultural Experiment Station in cooperation with U.S. Dept. of Agric Bull 556:565-599

20. Koenig R, Huth W (2000) Soilborne rye mosaic and European wheat mosaic virus: two names for a furovirus with variable genome properties which are widely distributed in several cereal crops in Europe. Arch Virol 145:689-697

21. Koenig R, Huth W (2003) Natural infection of wheat by the type strain of Soilborne wheat mosaic virus in New Zealand. Australas Plant Pathol 38:85-90

22. Kosambi DD (1944) The estimation of map distances from recombination values. Ann Eugen $12: 172-175$

23. Krill AM, Kirst M, Kochian LV, Buckler ES, Hoekenga OA (2010) Association and linkage analysis of aluminum tolerance genes in maize. PLoS One 5:e9958.

24. Kulwal P, Ishikawa G, Benscher D, Feng Z, Yu L, Jadhav A, Mehetre S, Sorrells ME (2012) Association mapping for pre-harvest sprouting resistance in white winter wheat. Theor Appl Genet 125:793-805 
25. Kump KL, Bradbury PJ, Wisser RJ, Buckler ES, Belcher AR, et al. (2011) Genome-wide association study of quantitative resistance to southern leaf blight in the maize nested association mapping population. Nat Genet 43:163-168

26. Lebas BSM, Ochoa-Corona FM, Elliott DR, Tang J, Blouin AG, Timudo OE, Ganev S, Alexander BJR (2009) Investigation of an outbreak of Soil-borne wheat mosaic virus in New Zealand. Australas Plant Pathol 38:85-90

27. Letta T, Maccaferri M, Badebo A, Ammar K, Ricci A, Crossa J, Tuberosa R (2013) Searching for novel sources of field resistance to Ug99 and Ethiopian stem rust races in durum wheat via association mapping. Theor Appl Genet 126:1237-1256

28. Liu S, Cai S, Graybosch R, Chen C, Bai G (2008) Quantitative trait loci for resistance to preharvest sprouting in U.S. hard white winter wheat Rio Blanco. Theor Appl Genet 117: 691699

29. Maccaferri M, Ratti C, Rubies-Autonell C, Vallega V, Demontis A, et al. (2011) Resistance to Soil-borne cereal mosaic virus in durum wheat is controlled by a major QTL on chromosome arm 2BS and minor loci. Theor Appl Genet 123:527-544

30. Malosetti M, van der Linden CG, Vosman B, van Eeuwijk FA (2007) A mixed-model approach to association mapping using pedigree information with an illustration of resistance to Phytophthora infestans in potato. Genetics 175:879-889.

31. McKinney HH (1923) Investigations on the rosette disease of wheat and its control. J Agric Res 23:771-800

32. Merkle OG, and Smith EL (1983) Inheritance of resistance to soilborne mosaic in wheat. Crop Sci 23:1075-1076.

33. Miyake M (1938) Mendelian inheritance of resistance to mosaic disease in wheat varieties. Jpn J Genet 14:239-2 
34. Modawi RS, Heyne EG, Brunetta D, Willis WG (1982) Genetic studies of field reaction to wheat Soilborne mosaic virus. Plant Dis 66:1183-1184.

35. Myers LD, Sherwood JL, Siegerist WC, Hunger RM (1993) Temperature-influenced virus movement in expression of resistance to Soilborne wheat mosaic virus in hard red winter wheat (Triticum aestivum). Phytopathology 83:548-551

36. Myles S, Peiffer J, Brown PJ, Ersoz ES, Zhang Z, et al. (2009) Association mapping: critical considerations shift from genotyping to experimental design. Plant Cell 21:2194-2202

37. Narasimhamoorthy B, Gill BS, Fritz AK, Nelson JC, and Brown- Guedira GL (2006) Advanced backcross QTL analysis of a hard winter wheat $\mathrm{x}$ synthetic wheat population. Theor Appl Genet 112:787-796.

38. Nijman IJ, Kuipers S, Verheul M, Guryev V, Cuppen E (2008) A genome-wide SNP panel for mapping and association studies in the rat. BMC Genomics 9:95

39. Perovic D, Förster J, Devaux P, Hariri D, Guilleroux M, et al. (2009) Mapping and diagnostic marker development for Soil-borne cereal mosaic virus resistance in bread wheat. Mol Breed 23:641-653

40. Pritchard JK, Stephens M, Donnelly PJ (2000) Inference of population structure using multilocus genotype data. Genetics 155:945-959

41. Reif JC, Gowda M, Maurer HP, Longin CFH, Korzun K (2011) Association mapping for quality traits in soft winter wheat. Theor Appl Genet (2011) 122:961-970

42. Sawada E (1927) Control of Wheat yellow mosaic virus. J Plant Prot 14:444-449

43. Semagn K, Babu R, Hearne S, Olsen M (2013) Single nucleotide polymorphism genotyping using Kompetitive Allele Specific PCR (KASP): overview of the technology and its application in crop improvement. Mol Breed DOI 10.1007/s1 1032-013-9917-x 
44. Shaalan MI, Heyne EG, and Sill WH (1966) Breeding wheat for resistance to Soil-borne wheat mosaic virus, Wheat streak-mosaic virus, leaf rust, stem rust, and bunt. Phytopathology 56:664-669.

45. Shirako Y, Suzuki N, French RC (2000) Similarity and divergence among viruses in the genus Furovirus. Virology 270:201-207

46. Somers DJ, Isaac P, Edwards K (2004) A high-density wheat microsatellite consensus map for bread wheat (Triticum aestivum L.) Theor Appl Genet 109:1105-1114

47. Terracciano I, Maccaferri M, Bassi F, Mantovani P, Sanguineti MC, et al. (2013) Development of COS-SNP and HRM markers for high throughput and reliable haplotypebased detection of Lr14a in durum wheat (Triticum durum Desf.). Theor Appl Genet, $126: 1077-1101$

48. Toojinda, T., E. Baird, A. Booth, L. Broers, and P. Hayes. 1998. Introgression of quantitative trait loci (QTLs) determining stripe rust resistance in barley: an example of marker-assisted line development. Theor Appl Genet 96:123-131

49. Van Ooijen JW, Voorrips RE (2001) JionMap version 3.0: Software for the calculation of genetic linkage maps

50. Zhang D, Bai G, Hunger RM, Bockus WW, Yu J, Carver BF and Brown-Guedira G (2011) Association study of resistance to Soilborne wheat mosaic virus in U.S. winter wheat. Phytopathology 101:1322-9.

51. Zhang D, Bai G, Zhu C, Yu J, Carver BF (2010) Genetic diversity, population structure, and linkage disequilibrium in U.S. elite winter wheat. Plant Gen 3:117-127.

52. Zhang XH, Pan HY, Bai GH (2012) Quantitative trait loci for Fusarium head blight resistance in U.S. hard winter wheat cultivar 'Heyne'. Crop Sci. doi: 10.2135/cropsci2011.08.0418 
53. Zhu C, Gore M, Buckler E, Yu J (2008) Status and prospects of association mapping in plants.

Plant Gen 1: 5-20. 
Figure legends:

Fig. 1 Number of wheat accessions in response to Soil-borne wheat mosaic virus infection. A, 205 accessions evaluated in spring 2010 and 2011 at Manhattan, KS. B, RILs from 'Trego' x 'Heyne' population. $\mathrm{R}=$ resistant, $\mathrm{MR}=$ moderate resistant, $\mathrm{MS}=$ moderate susceptible and $\mathrm{S}=$ susceptible.

Fig. 2 The distribution of '- $\log 10$ ( $P$-value)' by scanning 6985 single nucleotide polymorphism markers against two-seasons of field data for Soil-borne wheat mosaic virus (SBWMV) ratings and the mean over both seasons. A previously reported marker (Xgwm469) associated with SBWMV resistance is also listed as a control.

Fig. 3 SBWMV-resistance gene (Sbwm-1) was detected across all environments based on the linkage map of chromosome 5D developed from the 'Trego' $x$ 'Heyne' RIL population (A) and the syntenic region in barley (B), Brachypodium (C) and rice (D).

Supplemental Fig. 1 Linkage disequilibrium analysis indicated the six SNPs were within a strong linkage block with the marker Xgwm469 on wheat chromosome 5DL. The linkage map from Somers et al. (2004) was used as a reference map to indicate that only Xgwm469 had LD with the six SNPs, not other two adjacent markers (Xcfd10 and Xgwm565).

Supplemental Fig. 2 KASP assay profiling of SNP wsnp_CAP11_c209_198467 (A) and SNP wsnp_JD_c4438_5568170 (B) in 205 wheat accessions. (A). Allele X (KASPFAM, blue color) represents the A nucleotide, Allele Y (KASPHEX, green color) represents the G nucleotide. (B). Allele X (KASPFAM, blue color) represents the A nucleotide, Allele Y (KASPHEX, green color) represents the G nucleotide. The black dots and crosses in the circle represent water controls and missing data. 


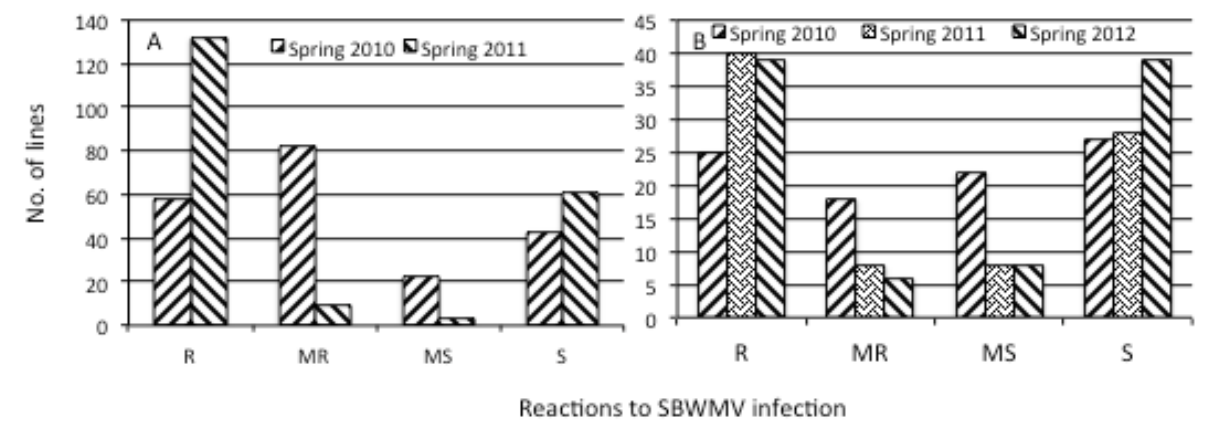

Fig. 1 


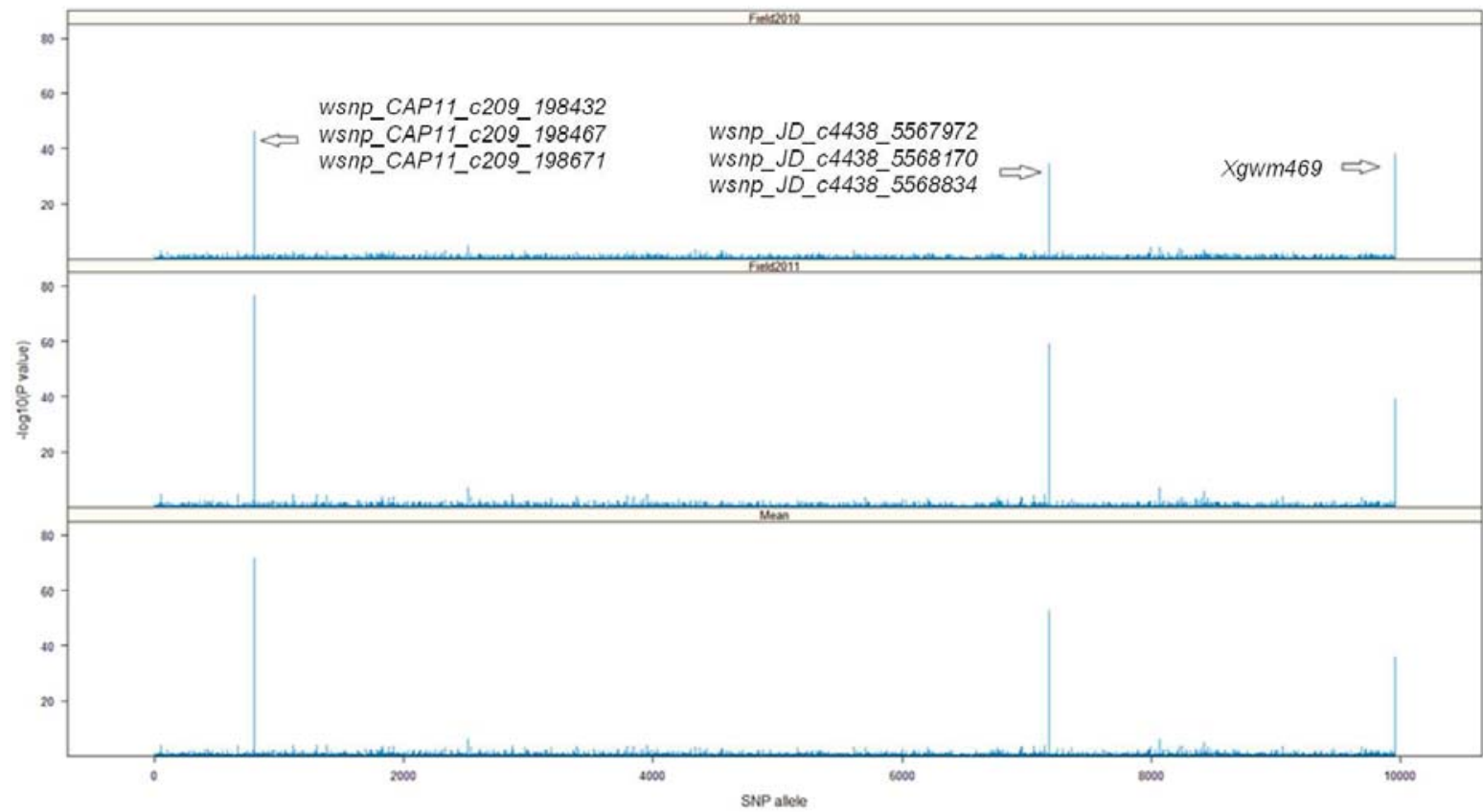

Fig. 2 
A

$5 \mathrm{D}$

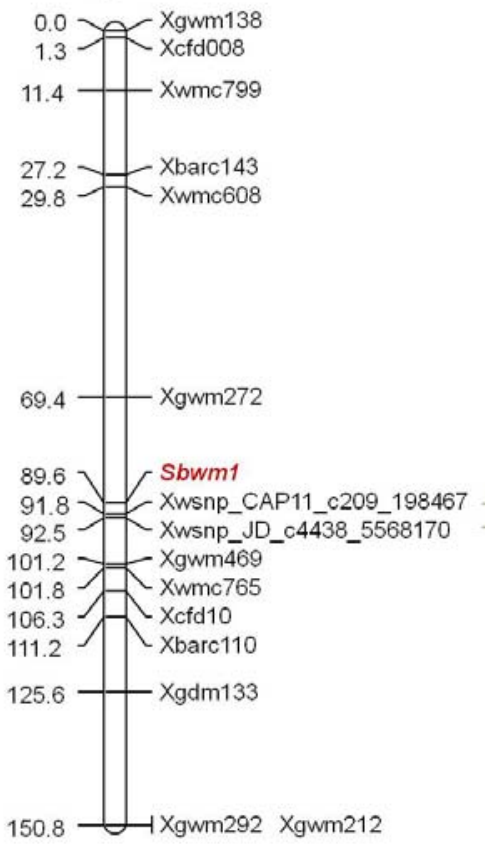

B

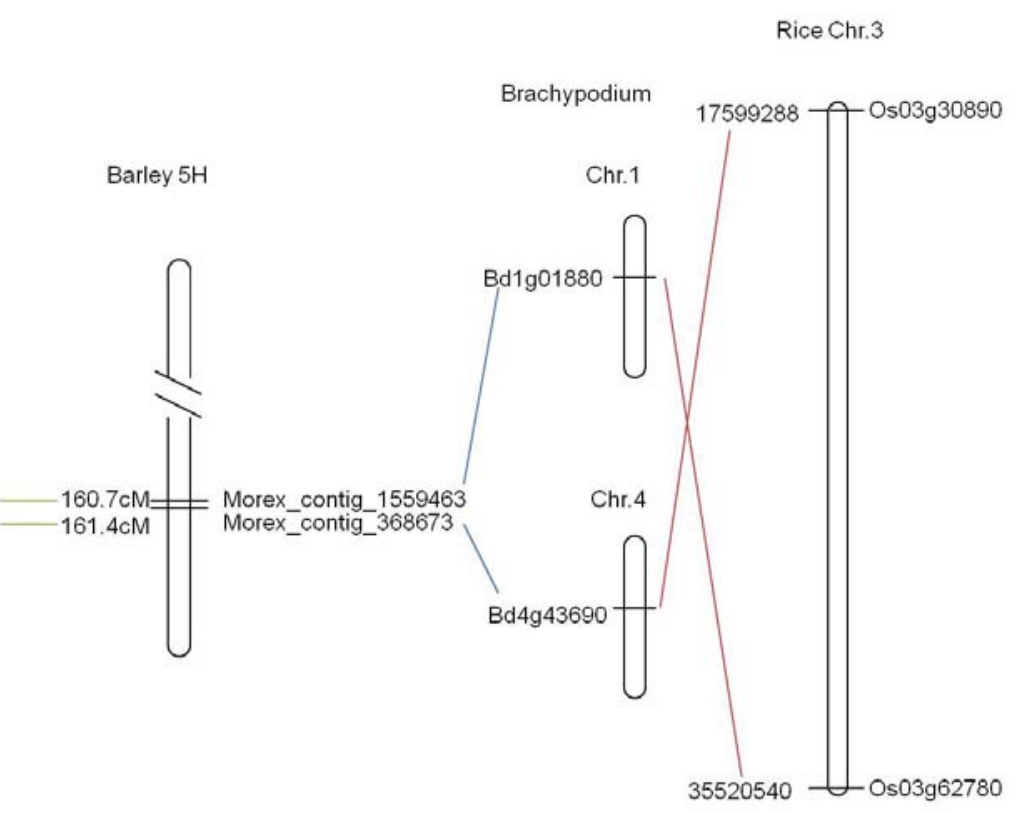

Fig. 3 


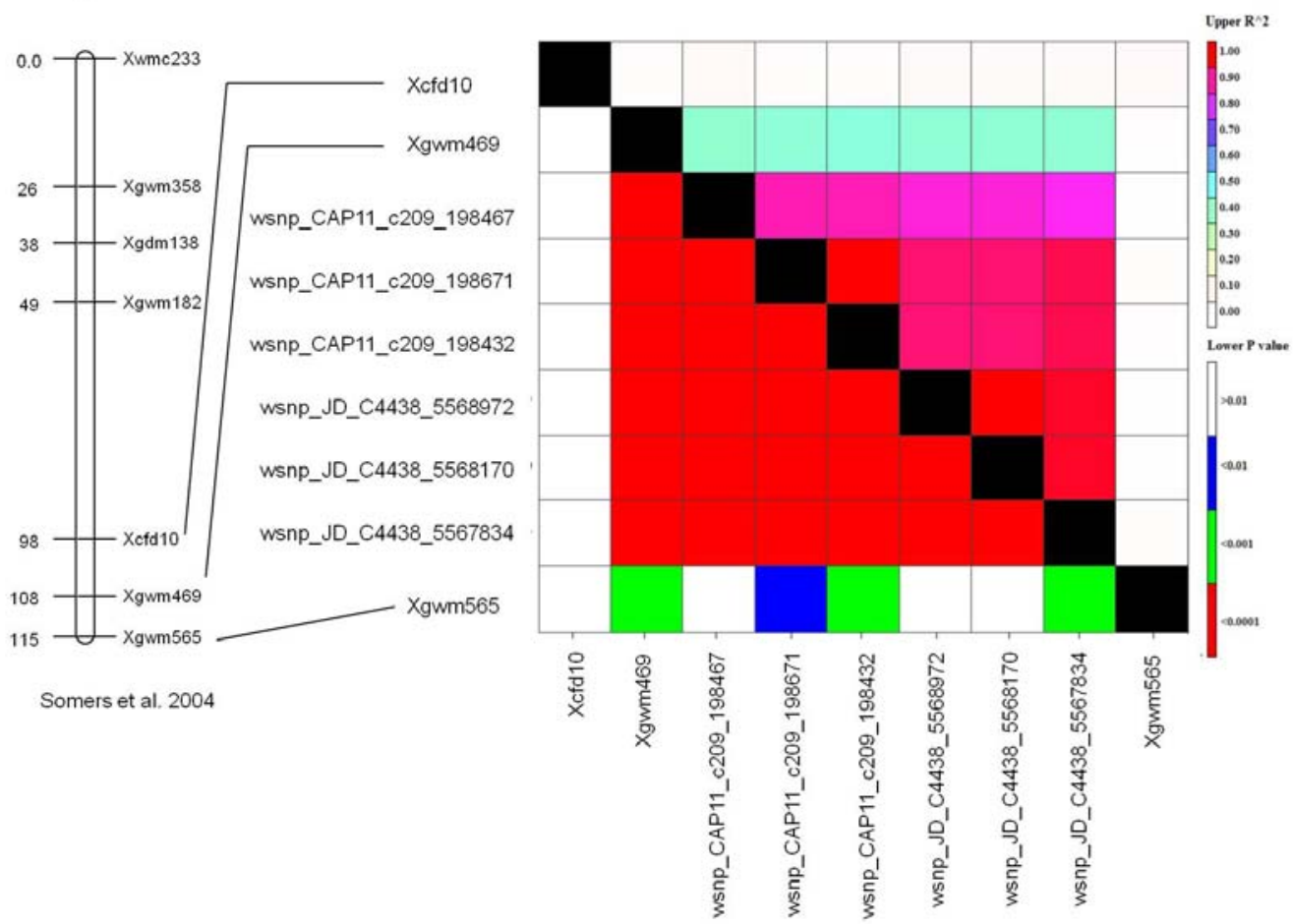

Supplemental Fig. 1 

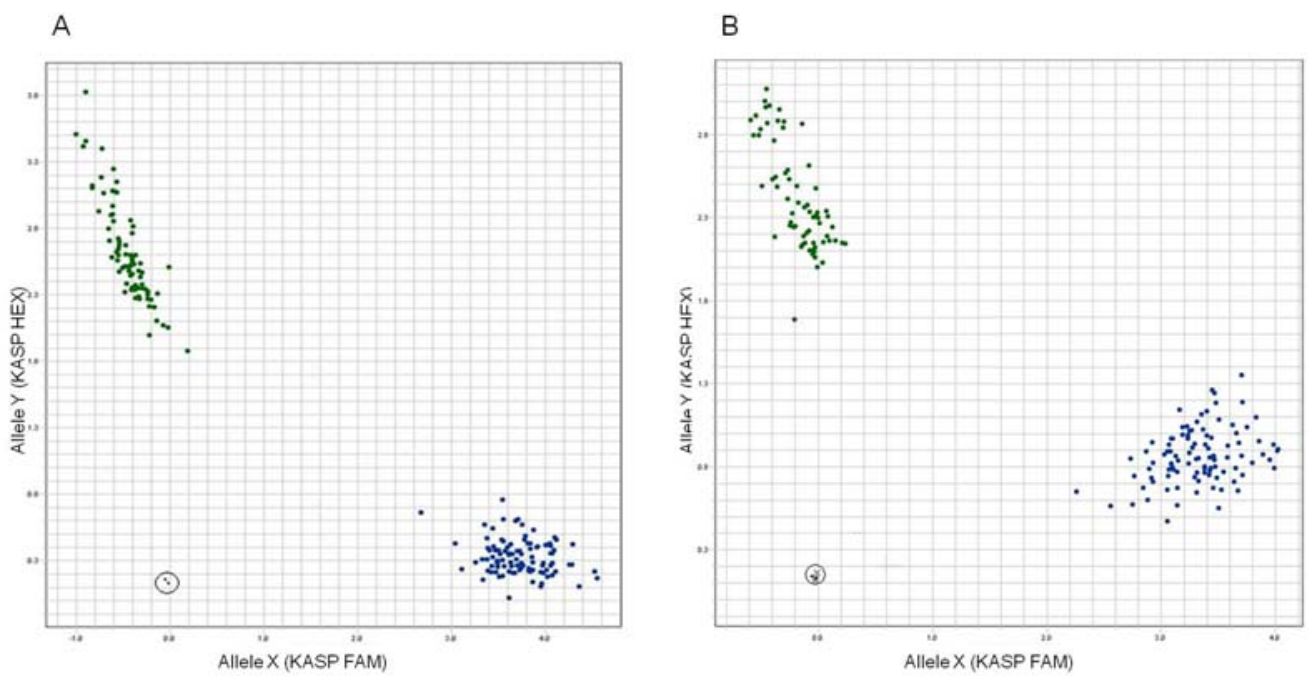

Supplemental Fig. 2 
Table 1 Haplotypes of the two SNPs significantly associated with Soil-borne wheat mosaic virus resistance in 205 wheat accessions

\begin{tabular}{lllll}
\hline Haplotypes & wsnp_CAP11_c209_198467 & wsnp_JD_c4438_5568170 & Accession & SBWMV \\
& & & No. & resistance \\
\hline R1 & A & A & 137 & Resistant \\
R2 & A & G & 5 & Resistant \\
S1 & G & G & 60 & Susceptible \\
S2 & G & A & 3 & Susceptible \\
\hline
\end{tabular}

